\title{
Insulating state and the importance of the spin-orbit coupling in $\mathrm{Ca}_{3} \mathrm{CoRhO}_{6}$
}

\author{
Hua Wu, Z. Hu, D. I. Khomskii, and L. H. Tjeng \\ II. Physikalisches Institut, Universität zu Köln, Zülpicher Str. 77, D-50937 Köln, Germany
}

\begin{abstract}
We have carried out a comparative theoretical study of the electronic structure of the novel onedimensional $\mathrm{Ca}_{3} \mathrm{CoRhO}_{6}$ and $\mathrm{Ca}_{3} \mathrm{FeRhO}_{6}$ systems. The insulating antiferromagnetic state for the $\mathrm{Ca}_{3} \mathrm{FeRhO}_{6}$ can be well explained by band structure calculations with the closed shell high-spin $d^{5}$ $\left(\mathrm{Fe}^{3+}\right)$ and low-spin $t_{2 g}^{6}\left(\mathrm{Rh}^{3+}\right)$ configurations. We found for the $\mathrm{Ca}_{3} \mathrm{CoRhO}_{6}$ that the Co has a strong tendency to be $d^{7}\left(\mathrm{Co}^{2+}\right)$ rather than $d^{6}\left(\mathrm{Co}^{3+}\right)$, and that there is an orbital degeneracy in the local Co electronic structure. We argue that it is the spin-orbit coupling which will lift this degeneracy thereby enabling local spin density approximation + Hubbard U (LSDA+U) band structure calculations to generate the band gap. We predict that the orbital contribution to the magnetic moment in $\mathrm{Ca}_{3} \mathrm{CoRhO}_{6}$ is substantial, i.e. significantly larger than $1 \mu_{B}$ per formula unit. Moreover, we propose a model for the contrasting intra-chain magnetism in both materials.
\end{abstract}

PACS numbers: 71.20.-b, 71.70.-d, 75.25.+z, 75.30.Gw

\section{INTRODUCTION}

The synthesis of the one-dimensional cobaltate $\mathrm{Ca}_{3} \mathrm{Co}_{2} \mathrm{O}_{6} \frac{1,2}{2}$ and the discovery of the exotic stair-step jumps in the magnetization at regular intervals of the applied magnetic field ${ }^{3}, 4$ have triggered a flurry of research activities, both experimentally $\underline{4}, 5,6,7,8,9,10,11,12,13,14,15,16$ and theoretically, $\stackrel{17,18,19,20,21,22,23,24}{1}$ This compound consists of $\left[\mathrm{Co}_{2} \mathrm{O}_{6}\right]_{\infty}$ chains running along the $c$ axis of the hexagonal unit cell, with in each chain alternating $\mathrm{CoO}_{6}$ octahedra and $\mathrm{CoO}_{6}$ trigonal prisms. $\frac{1}{2}$ The intra-chain coupling is ferromagnetic (FM) with a Curie temperature of $T_{C}=24 \mathrm{~K}$ and the inter-chain antiferromagnetic (AF) with a Néel temperature of $T_{N}=10 \mathrm{~K} \stackrel{\underline{3}}{\text { Owing }}$ to an inter-chain magnetic frustration associated with the triangular lattice, the magnetic ground-state is either a partially disordered $\mathrm{AF}$ state or a spin-freezing state ${ }^{3}$ Substitution of the Co by other transition metals results in quite dramatic changes of the properties. The $\mathrm{Ca}_{3} \mathrm{CoRhO}_{6}$ compound has its $T_{C}$ shifts up to a high value of $90 \mathrm{~K}$ and $T_{N}$ to $35 \mathrm{~K}, 25,26,27,28,29,30$ but the type of its magnetic ground-state is similar to $\mathrm{Ca}_{3} \mathrm{Co}_{2} \mathrm{O}_{6}, \stackrel{26,27}{2}$ while $\mathrm{Ca}_{3} \mathrm{FeRhO}_{6}$ is an intra-chain $\mathrm{AF}$ with $T_{N}=12 \mathrm{~K} \cdot 25,29,31$ In these compounds the Rh ions occupy the octahedral sites while the $\mathrm{Co} / \mathrm{Fe}$ reside within the trigonal prisms $\stackrel{25,29}{\underline{2}}$

There is a debate about the electronic structure of these one-dimensional cobaltates in the literature, in particular for

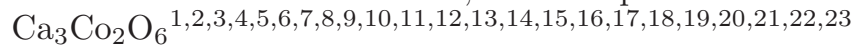
and $\mathrm{Ca}_{3} \mathrm{CoRhO}_{6}, 16,17,25,26,27,28,29,30,32$ Consensus has yet to arrive about the valence, spin and orbital state of the Co ions, without which one could not make a reliable modeling of the magnetic properties. Focusing on the $\mathrm{Ca}_{3} \mathrm{CoRhO}_{6}$ system, it was originally thought that the valence state is $\mathrm{Co}^{2+} / \mathrm{Rh}^{4+}$ based on magnetization measurements and bond valence sum. ${ }^{25,26}$ Subsequent neutron studies concluded, however, that the $\mathrm{Co}^{3+} / \mathrm{Rh}^{3+}$ scenario is a better description. 27,30 Very recently, a photoemission investigation favors yet the original $\mathrm{Co}^{2+} / \mathrm{Rh}^{4+}$ assignment $\underline{16}$ Existing band structure calculations also provide a mixed message: a generalized-gradient-approximation (GGA) study suggests the $\mathrm{Co}^{3+} / \mathrm{Rh}^{3+}$ state $\stackrel{17}{\longleftarrow}$ while an LSDA calculation leaves this issue open ${ }^{32}$ Important is to note that both calculations predict $\mathrm{Ca}_{3} \mathrm{CoRhO}_{6}$ to be a metal, in strong disagreement with the experiment $\underline{10,16}$

To resolve these disagreements, we carried out a theoretical study of the electronic structure and magnetic properties of $\mathrm{Ca}_{3} \mathrm{CoRhO}_{6}$ in which we took the insulating state of the material 10,16 as a key finding. For this we applied the LSDA+U method 33,34 in order to take into account more explicitly the correlated motion of the electrons typical for transition metal oxides. Using $\mathrm{Ca}_{3} \mathrm{FeRhO}_{6}$ as a reference, we find that the Co ions have a lower valence than $\mathrm{Fe}$, and that the $\mathrm{Co}$ are in an orbitally degenerate state while the $\mathrm{Fe}$ are not. The valence assignment coming from our calculations is the $\mathrm{Fe}^{3+} / \mathrm{Rh}^{3+}$ for $\mathrm{Ca}_{3} \mathrm{FeRhO}_{6}$ and $\mathrm{Co}^{2+} / \mathrm{Rh}^{4+}$ for $\mathrm{Ca}_{3} \mathrm{CoRhO}_{6}$. The insulating state for $\mathrm{Ca}_{3} \mathrm{FeRhO}_{6}$ can be readily reproduced, but for $\mathrm{Ca}_{3} \mathrm{CoRhO}_{6}$ we infer that the spin-orbit coupling (SOC) must be included in the LSDA+U scheme. We make a testable prediction, namely that there is a large orbital contribution to the magnetic moment in $\mathrm{Ca}_{3} \mathrm{CoRhO}_{6}$, about $1.7 \mu_{B}$ per formula unit. We also calculate the various exchange constants and propose a model to explain the weak intrachain $\mathrm{AF}$ in $\mathrm{Ca}_{3} \mathrm{FeRhO}_{6}$ and enhanced intra-chain $\mathrm{FM}$ in $\mathrm{Ca}_{3} \mathrm{CoRhO}_{6}$.

\section{RESULTS AND DISCUSSION}

Our calculations are performed by using the fullpotential augmented plane waves plus local orbital method ${ }^{35}$ We took the crystal structure data determined by $\mathrm{x}$-ray diffraction 25 The muffin-tin sphere radii are chosen to be 2.7, 2.0 and 1.6 Bohr for $\mathrm{Ca}, \mathrm{Co} / \mathrm{Fe} / \mathrm{Rh}$, and $\mathrm{O}$ atoms, respectively. The cut-off energy of $16 \mathrm{Ryd}$ is used for plane wave expansion of interstitial wave functions, and $5 \times 5 \times 5 \mathrm{k}$-mesh for integrations over the Brillouin zone, both of which ensuring a sufficient numeri- 


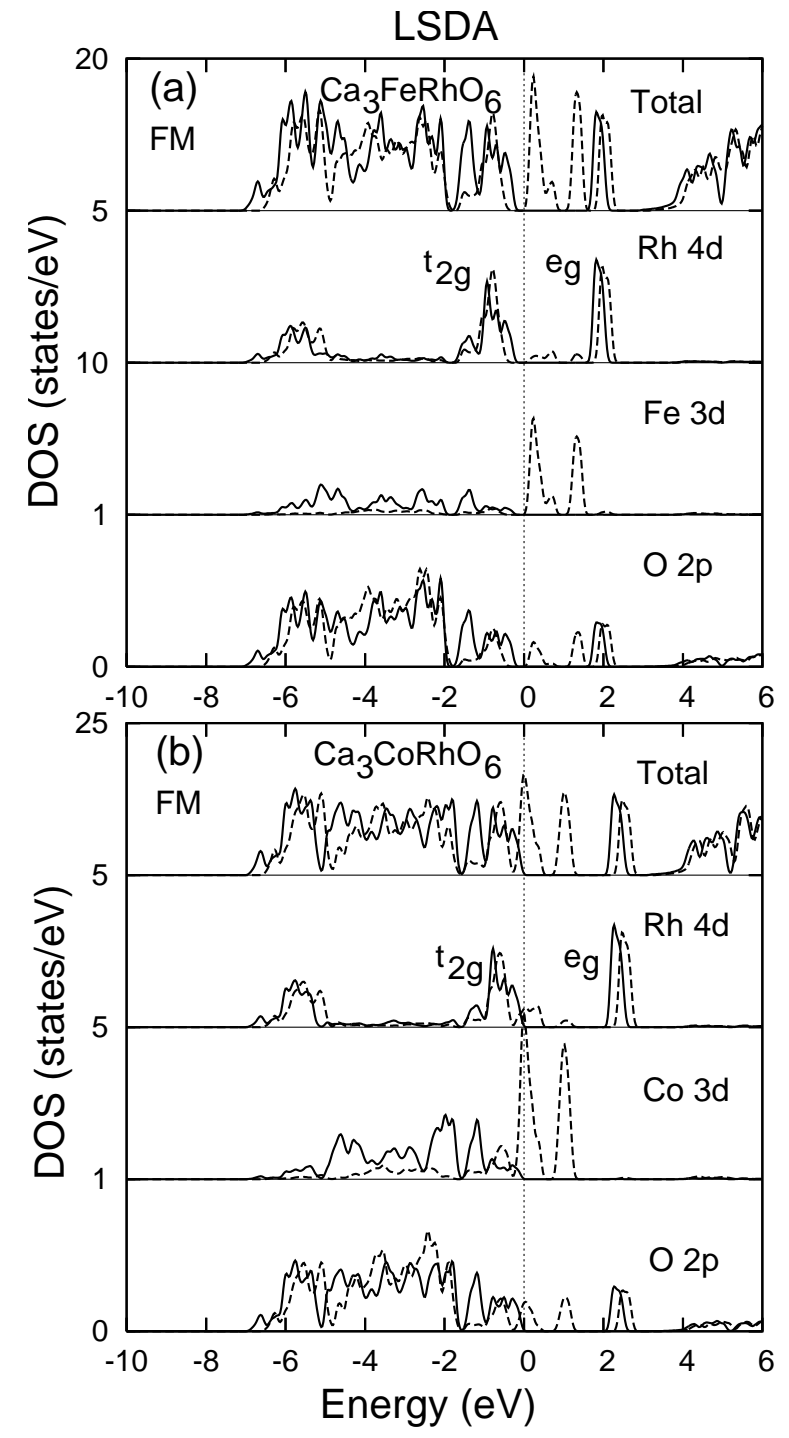

FIG. 1: Density of states (DOS) of $\mathrm{Ca}_{3} \mathrm{FeRhO}_{6}$ (panel a) and $\mathrm{Ca}_{3} \mathrm{CoRhO}_{6}$ (panel b) in the ferromagnetic (FM) state from LSDA. The Fermi level is set at zero energy. Both panels show, from top to bottom, the total DOS, the Rh $4 d$, Fe/Co $3 d$, and O $2 p$ partial DOS. Solid (dashed) lines depict the spin-up (down) states.

cal accuracy. The SOC is included by second-variational method with the scalar relativistic wave functions,,$\frac{35}{5}$ and actually $l_{z}$ and $s_{z}$ are good quantum numbers due to the special trigonal crystal field of the Co sites as seen below. The easy magnetization direction is along the $c$-axis chains. In view of those $c$-axis chains being well separated in the hexagonal $a b$-plane and of these two compounds behaving like a quasi-one-dimensional system, we study in this work the type and origin of the intra-chain magnetic coupling, thereby assuming a FM inter-chain coupling.

As a reference, we first calculate the electronic structure of $\mathrm{Ca}_{3} \mathrm{FeRhO}_{6}$ in the FM state using the LSDA. Fig. 1(a) shows the density of states (DOS). One can clearly see that the octahedral $\mathrm{Rh} 4 d$ has a large $t_{2 g^{-}}$ $e_{g}$ crystal-field $(\mathrm{CF})$ splitting of about $3 \mathrm{eV} \stackrel{36}{\underline{3}}$ With the $t_{2 g}$ shell completely full and the $e_{g}$ completely empty, the $\mathrm{Rh}$ is formally $3+\left(4 d^{6}\right)$ and takes the low-spin (LS, $S=0)$ state. One can also observe that the Fe $3 d$ spin-up states are essentially completely full and the spin-down completely empty. The Fe is thus formally $3+\left(3 d^{5}\right)$ and high-spin (HS, $S=5 / 2$ ). The Fe partial DOS also shows that the unoccupied spin-down states are split roughly into two groups with about $1 \mathrm{eV}$ separation. This is caused by the presence of a trigonal $\mathrm{CF}$, making $\left(d_{1}\right.$, $\left.d_{-1}\right)$ orbitals to lie higher than the nearly degenerate $\left(d_{0}, d_{2}, d_{-2}\right), 2,17,18,19,20,21,22,23$ Covalency reduces the $\mathrm{Fe}$ moment to $3.73 \mu_{B}$ but creates at the same time 0.20 $\mu_{B}$ moment at the Rh and $0.13 \mu_{B}$ at each $\mathrm{O}$, so that the total moment is still $5.00 \mu_{B}$, see Table I. The material is an insulator, since it is effectively a closed shell system due to the sufficiently large CF splitting at the $\mathrm{Rh}$ and exchange splitting on the Fe. We have also calculated the $\mathrm{Ca}_{3} \mathrm{FeRhO}_{6}$ in the intra-chain $\mathrm{AF}$ state, and found very similar DOS'ses (the spin part not considered). Important is that the total energy of the $\mathrm{AF}$ is lower than the FM by about $11 \mathrm{meV}$ (see Table I), explaining why $\mathrm{Ca}_{3} \mathrm{FeRhO}_{6}$ is an AF. Our LSDA finding is in close agreement with the GGA results of Villesuzanne and Whangbo $\stackrel{22}{\underline{2}}$

Fig. 1(b) depicts the LSDA results for the $\mathrm{Ca}_{3} \mathrm{CoRhO}_{6}$ in the FM state. The DOS shows quite a number of similarities with that of the $\mathrm{Ca}_{3} \mathrm{FeRhO}_{6}$, including the large $\mathrm{CF}$ at the $\mathrm{Rh}$ and the characteristic trigonal $\mathrm{CF}$ splitting at the $\mathrm{Fe} / \mathrm{Co}$. The only significant difference is the fact that the Co $3 d$ lies lower in energy than the Fe $3 d$, which is reasonable since Co is less electropositive than Fe. The consequences are, however, quite dramatic: the Fermi level now straddles through the $\left(d_{0}, d_{2}, d_{-2}\right)$ part of the Co $3 d$ spin down band, meaning that $\mathrm{Ca}_{3} \mathrm{CoRhO}_{6}$ would be a metal. This LSDA result is in clear disagreement with experimental observations $\underline{10,16}$ Also Whangbo et al $\stackrel{17}{ }$ found a metallic solution. We have investigated the intra-chain AF state for $\mathrm{Ca}_{3} \mathrm{CoRhO}_{6}$ and found that it is also metallic. It has, however, a higher energy than the FM by about $106 \mathrm{meV}$, see Table I. This means that system tends to be FM, at least if one is willing to trust these LSDA results as far as the magnetism is concerned. It is obvious, nevertheless, that this half-metallic solution is an artifact of the LSDA.

The LSDA of $\mathrm{Ca}_{3} \mathrm{CoRhO}_{6}$ shows that the Fermi level is located in a narrow Co $3 d$ band with no more than $1 \mathrm{eV}$ width. It is then also natural to expect that already modest electron correlation effects at the Co sites will have a pronounced effect on the energetics of this band. We therefore carried out LSDA+U band structure calculations ${ }^{34}$ for both the $\mathrm{Ca}_{3} \mathrm{CoRhO}_{6}$ and $\mathrm{Ca}_{3} \mathrm{FeRhO}_{6}$ with the Coulomb energy $U$ is set to 5,4 , and $3 \mathrm{eV}$, and the Hund's exchange parameter $J_{H}$ to $0.9,0.9,0.5 \mathrm{eV}$ for the Co $3 d, \mathrm{Fe} 3 d$, and Rh $4 d$ shells, respectively.

We will first discuss the results for the $\mathrm{Ca}_{3} \mathrm{FeRhO}_{6}$, our reference system. Fig. 2(a) shows the LSDA+U DOS of 


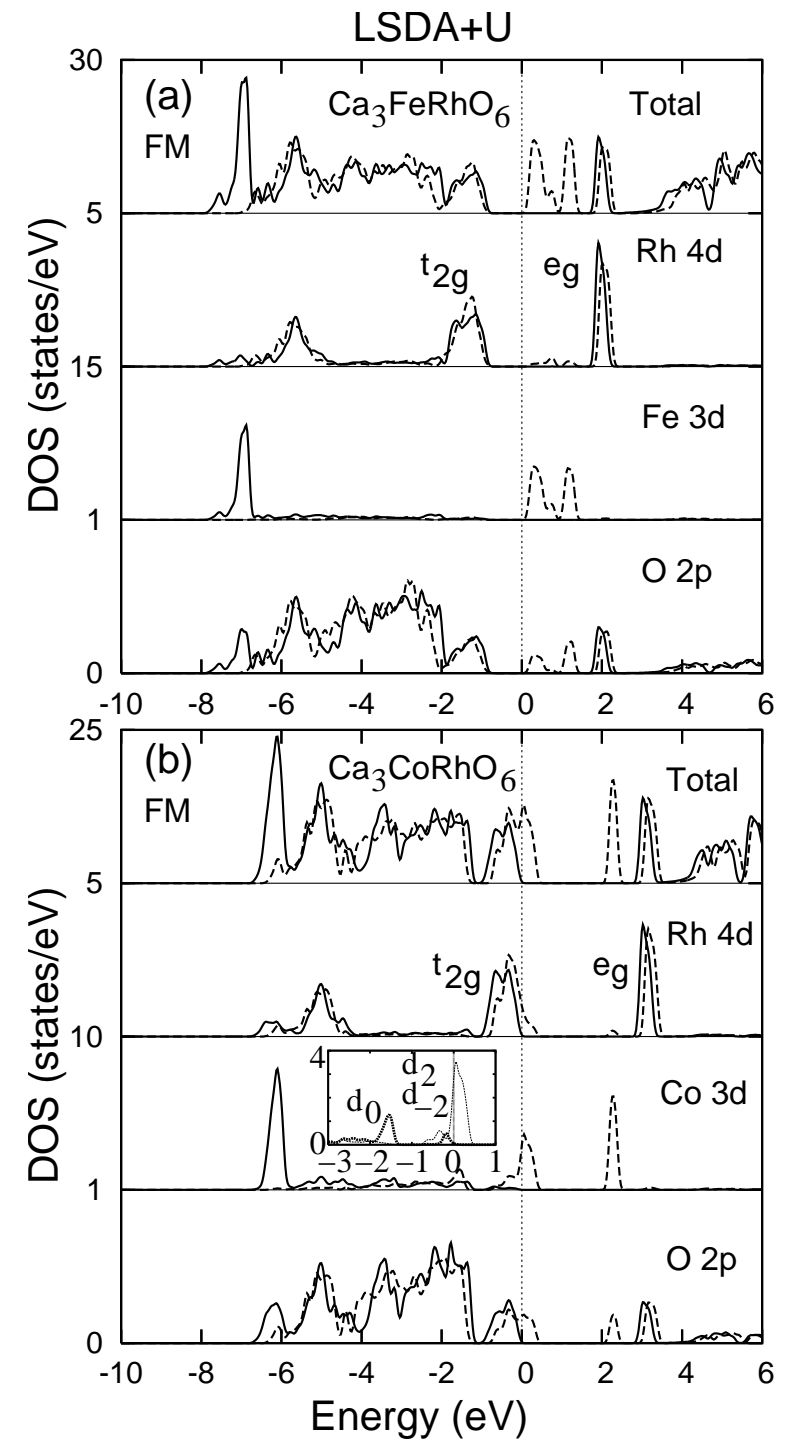

FIG. 2: Density of states (DOS) of $\mathrm{Ca}_{3} \mathrm{FeRhO}_{6}$ (panel a) and $\mathrm{Ca}_{3} \mathrm{CoRhO}_{6}$ (panel b) in the ferromagnetic (FM) state from LSDA+U. Inset: close up of the down-spin Co $d_{0}, d_{2}$ and $d_{-2}$ DOS. See Fig. 1 caption for other notations.

$\mathrm{Ca}_{3} \mathrm{FeRhO}_{6}$ in the FM state. One can observe that the energy separation between the fully occupied $t_{2 g}$ shell and the fully unoccupied $e_{g}$ has increased in comparison with the LSDA results [Fig. 1(a)], in accordance with the inclusion of $U$. This also happens with the separation between Fe $3 d$ spin-up and spin-down states, whereby the spin-up states are pushed down to about $-7 \mathrm{eV}$. This deep-lying state reflects the high stability typical for $3 d^{5}$ ions, in which the high-spin configuration of the half-filled shell gives an energy gain of $4 J_{H}$. With the Fe spin-up states being at the bottom of the valence band, the covalency with the $\mathrm{Rh} 4 d$ and $\mathrm{O} 2 p$ bands is much reduced. This is then also reflected in the increase (with respect to LSDA) of the Fe moment to $3.95 \mu_{B}$ and decrease of the $\mathrm{Rh}$ to $0.11 \mu_{B}$ and of each $\mathrm{O}$ to $0.11 \mu_{B}$. The total magnetic moment should not change: it remains indeed at $5.00 \mu_{B}$, see table I. As far as the low energy scale physics is concerned, the band gap has increased from about $0.2 \mathrm{eV}$ to an appreciable $0.9 \mathrm{eV}$. It does not directly scale with the $U$ 's, since it is determined by the occupied Rh $t_{2 g}$ and the unoccupied Fe $3 d$ spin-down states. Note that our conclusion that $\mathrm{Ca}_{3} \mathrm{FeRhO}_{6}$ is an insulator with $\mathrm{HS}-\mathrm{Fe}^{3+} / \mathrm{LS}-\mathrm{Rh}^{3+}$ and is a Heisenberg spin-chain system (see below), is $U$-independent.

Calculations for the intra-chain $\mathrm{AF}$ state of $\mathrm{Ca}_{3} \mathrm{FeRhO}_{6}$ give very similar DOS'ses (the spin part not considered), with practically the same total energy as the FM (the difference being about $2 \mathrm{meV}$ is within the error bar, see Table I). This and the above LSDA result indicate that the intra-chain AF exchange interaction is very weak. In this situation the effects not included in our calculations, such as the detailed type of inter-chain ordering (we assumed that spins in all neighboring chains are in phase, i.e., we considered FM inter-chain ordering) may start to play a role and may finally determine the type of long-range ordering in $\mathrm{Ca}_{3} \mathrm{FeRhO}_{6}$. Thus from the present LSDA+U calculations we can only conclude that the FM and $\mathrm{AF}$ intra-chain orderings in this system are practically degenerate. We present below (Section III) qualitative arguments that in reality most probably the intra-chain interaction in $\mathrm{Ca}_{3} \mathrm{FeRhO}_{6}$ is weak but AF. All this is not inconsistent with the rather small $T_{N}$ value of $12 \mathrm{~K}$.

The LSDA+U results for $\mathrm{Ca}_{3} \mathrm{CoRhO}_{6}$ are shown in Fig. 2(b). Similar to the $\mathrm{Ca}_{3} \mathrm{FeRhO}_{6}$ case, the inclusion of $U$ increases the splitting between the Rh $4 d t_{2 g}$ and $e_{g}$ orbitals as well as Co $3 d$ spin-up and spin-down bands. Also similar is the reduction of the covalency of the $3 d$ spin-up as it is shifted to very low energies. Surprisingly, however, the insulating state is not formed. The Fermi level still straddles through the lower-energy part of the Co $3 d$ spin-down band. The main influence of $U$ here is only that the effective crystal splitting with the $\left(d_{1}, d_{-1}\right)$ orbitals is increased. To investigate why there is no gap opening, we plot in the inset of Fig. 2(b) a close up of the down-spin Co $d_{0}, d_{2}$ and $d_{-2}$ states in the vicinity of the Fermi level. We can now observe that the spindown $d_{0}$ is fully occupied, and that it is the $d_{2}$ and $d_{-2}$ bands which are partially occupied. This means first of all, with the spin-up states fully occupied, that the Co ion is essentially in the $d^{7}$ or $2+$ valence state, and not in the $d^{6}$ or $3+$ as proposed recently $\underline{17,27,30}$ Secondly, the inset reveals that it is the degeneracy of the $d_{2}$ and $d_{-2}$ bands which makes the LSDA $+\mathrm{U}$ to be inoperative to open the gap. If the $d_{0}$ were higher in energy than the $d_{2}$ and $d_{-2}$, or if the Co valence were $3+$, then LSDA+U would certainly have produced a band gap. For completeness, we have also calculated the AF state with the LSDA+U, and found also a metallic state with a $13 \mathrm{meV}$ higher total energy, see Table I.

We propose to include the SOC to lift the $d_{2}, d_{-2}$ degeneracy. The use of the SOC to lift the degeneracy in correlated insulators has surprisingly been done only in a few instances $\stackrel{37,38}{ }$ In most cases, this has 
been omitted, even for materials for which it is known that the orbital contribution to the magnetic moment is substantial. $33,39,40,41,42$ We claim that the SOC issue is essential for this particular $\mathrm{Ca}_{3} \mathrm{CoRhO}_{6}$ compound, motivated also by the report that the magnetocrystalline anisotropy is significant. ${ }^{26}$ Note that for the $\mathrm{Co}^{2+}$ ions in $\mathrm{Ca}_{3} \mathrm{CoRhO}_{6}$, the $d_{0}$ and $d_{2} / d_{-2}$ states are split off from the higher-lying $d_{1} / d_{-1}$ states by about $1 \mathrm{eV}$ [Fig. 1(b)], i.e., much larger than the SOC energy scale of about $70 \mathrm{meV} \stackrel{43}{ }$ Therefore, the SOC Hamiltonian can be simplified into just $\zeta l_{z} s_{z}$ by neglecting the $l_{+} s_{-}$and $l_{-} s_{+}$ mixing terms, both of which cause a mixing between the $d_{0}$ and $d_{1} / d_{-1}$, or between the $d_{2}\left(d_{-2}\right)$ and $d_{1}\left(d_{-1}\right)$. Thus, the $l_{z}$ and $s_{z}$ are good quantum numbers in this particular case.

The results of the $\mathrm{LSDA}+\mathrm{U}+\mathrm{SOC}$ calculations for $\mathrm{Ca}_{3} \mathrm{CoRhO}_{6}$ in the intra-chain FM state are shown in Fig. 3. We now can observe that a gap has been opened near the Fermi level, consistent with the experimental finding that the system is an insulator 10,16 The band gap is small and is given mainly by the weakly split Rh $4 d$ bands. The system is a Mott-Hubbard insulator in which the $\mathrm{U}$ in the $\mathrm{Rh} 4 d$ shell is the determining factor $44 \mathrm{As}$ far as the Co $3 d$ states are concerned, the band gap is about $2 \mathrm{eV}$, i.e. so large that it no longer determines the band gap of the system. A clearer view is offered in the inset of Fig. 3 which depicts a close up of the down-spin Co $d_{0}, d_{2}$ and $d_{-2}$ states in the vicinity of the Fermi level. One can see that the spin-down $d_{2}$ band is now fully occupied and the $d_{-2}$ fully unoccupied, i.e. there is no partial occupation anymore like in the LSDA+U. Interesting is to note that the spin-down $d_{0}$ band has also an unoccupied component slightly above the Fermi level, but not at the Fermi level. This can be attributed to the band formation with the unoccupied $\mathrm{Rh} 4 d 3 z^{2}-r^{2}$ state (i.e. $a_{1 g}$ in the trigonal $\mathrm{CF}$ ), demonstrating the $\mathrm{Rh}^{4+}$ valence state with the $t_{2 g}^{5}$ configuration and the one-dimensional character along the $c$-axis chain.

While the approach to include the SOC is sound in view of the sizable magnetocrystalline anisotropy, 26 a definitive justification needs to come from a determination of the magnitude of the orbital contribution to the magnetic moment: our LSDA $+\mathrm{U}+\mathrm{SOC}$ calculations $(U=5 \mathrm{eV}$ for $\mathrm{Co})$ predict for the $\mathrm{Co}^{2+}$ ions not only a spin moment of $2.72 \mu_{B}$ but especially a very large orbital moment of $1.69 \mu_{B}$ along the $c$-axis chain. This is a testable prediction, which is actually confirmed by a recent x-ray magnetic circular dichroism study $\underline{45}$ We verified that both the calculated spin and orbital moments stay constant within $0.1 \mu_{B}$ when the $U$ for Co is varied in the range of 2.5-6 eV. This huge orbital moment and the SOC firmly fix the orientation of the total magnetic moment along the $c$-axis chain, and thus the magnetism of $\mathrm{Ca}_{3} \mathrm{CoRhO}_{6}$ is highly Ising-like. The calculations also predict an appreciable $0.54 \mu_{B}$ spin moment on the $\mathrm{Rh}^{4+}$ $4 d$, and $0.09 \mu_{B}$ on each oxygen, showing the effect of covalency. Note that the $\mathrm{Rh}^{4+}$ ions carry no orbital moment, because the one hole resides on the $a_{1 g}$ singlet split

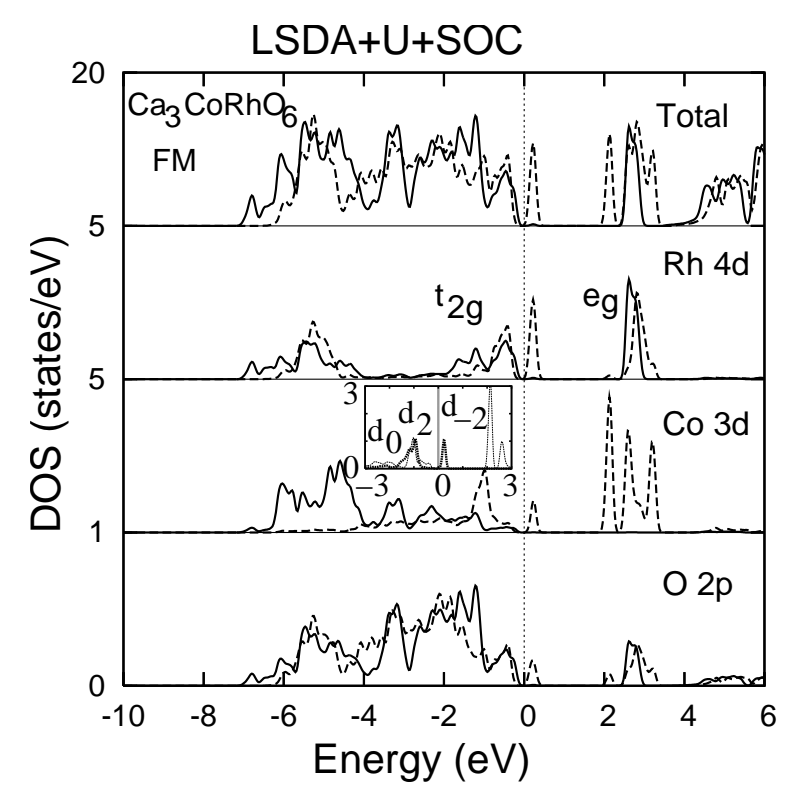

FIG. 3: Density of states (DOS) of $\mathrm{Ca}_{3} \mathrm{CoRhO}_{6}$ in the ferromagnetic (FM) state from LSDA+U+SOC. Inset: close up of the down-spin Co $d_{0}, d_{2}$ and $d_{-2}$ DOS. See Fig. 1 caption for other notations.

off from the $t_{2 g}$ manifold by the trigonal CF. We also note that the $\mathrm{Co}^{2+} / \mathrm{Rh}^{4+}$ valence state of $\mathrm{Ca}_{3} \mathrm{CoRhO}_{6}$, as we obtained in this work, remain unchanged by counting the electron occupation of the down-spin Co $d_{0}$ and $d_{2}$ orbitals (the up-spin Co $d$ shell is fully occupied) and the hole occupation of the down-spin Rh $d_{0}$ (i.e. $a_{1 g}$ ) orbital, once an insulating gap is opened at $U>2.5 \mathrm{eV}$ for $\mathrm{Co}$ and $U>2 \mathrm{eV}$ for $\mathrm{Rh}$ in our LSDA+U calculations including the spin-orbit coupling. All in all, our $\mathrm{Co}^{2+} / \mathrm{Rh}^{4+}$ so- $^{-}$ lution is thus consistent with the large total magnetic moment as measured by neutron diffraction. ${ }^{26,27,30}$

We also have carried out LSDA+U+SOC calculations for $\mathrm{Ca}_{3} \mathrm{CoRhO}_{6}$ in the intra-chain AF state, and we have found that this state is $70 \mathrm{meV}$ higher in energy than the FM state, see Table I. This allows us to estimate the effective FM exchange parameter of a $\mathrm{Co}^{2+}$ pair being 15 meV, using the simple Heisenberg model with the HS$\mathrm{Co}^{2+}$ spin-3/2. These numbers are substantially larger than for the $\mathrm{Ca}_{3} \mathrm{Co}_{2} \mathrm{O}_{6}$ system: there the AF-FM difference is $12 \mathrm{meV}$ and the exchange parameter about $1.5 \mathrm{meV}: 23$ Our calculations thus nicely explain why the intra-chain $T_{C}$ of $\mathrm{Ca}_{3} \mathrm{CoRhO}_{6}(90 \mathrm{~K})$ is much higher than that of $\mathrm{Ca}_{3} \mathrm{Co}_{2} \mathrm{O}_{6}(24 \mathrm{~K})$. For completeness, we have also carried out LSDA+U+SOC calculations for the $\mathrm{Ca}_{3} \mathrm{FeRhO}_{6}$. The results, however, are almost identical to those of the LSDA+U, since the SOC is not operative for the closed spin-up $\mathrm{Fe}^{3+} 3 d$ shell and the full $\mathrm{Rh}^{3+} t_{2 g}^{6}$ state. The absence of an orbital moment accounts for its Heisenberg spin character and very weak magnetocrystalline anisotropy 31 
TABLE I: Calculated electronic states of $\mathrm{Ca}_{3} \mathrm{FeRhO}_{6}$ and $\mathrm{Ca}_{3} \mathrm{CoRhO}_{6}(\mathrm{FM}$ : ferromagnetic; AF: antiferromagnetic; -I:insulator; -M:metal); total energy (meV) per formula unit relative to the ground state; spin moments $\left(\mu_{B}\right)$ at each Fe/Co, Rh, and $\mathrm{O}$ ion, as well as in the interstitial region and total magnetic moment per formula unit. Only the Co has the orbital moment and is shown. The Ca spin moment is about $0.01 \mu_{B}$ and the Fe orbital moment $0.02 \mu_{B}$, and are thus omitted.

\begin{tabular}{|c|c|c|c|c|c|c|c|c|c|}
\hline$\overline{\mathrm{Ca}_{3} \mathrm{FeRhO}_{6}}$ & state & energy & $\overline{\mathrm{Fe}}$ & & $\overline{\mathrm{Rh}}$ & $\overline{\mathrm{O}}$ & interstitial & total & Figure \\
\hline$\overline{\mathrm{LSDA}}$ & FM-I & 11 & 3.73 & & 0.20 & 0.13 & 0.27 & 5.00 & Fig. 1(a) \\
\hline LSDA & AF-I & 0 & \pm 3.72 & & 0 & \pm 0.12 & 0 & 0 & \\
\hline$\overline{\mathrm{LSDA}+\mathrm{U}}$ & FM-I & 0 & 3.95 & & 0.11 & 0.11 & 0.23 & 5.00 & Fig. 2(a) \\
\hline $\mathrm{LSDA}+\mathrm{U}$ & AF-I & 2 & \pm 3.95 & & 0 & \pm 0.11 & 0 & 0 & \\
\hline$\overline{\mathrm{Ca}_{3} \mathrm{CoRhO}_{6}}$ & state & energy & $\mathrm{Co}$ & $\mathrm{Co}^{\text {orb }}$ & $\overline{\mathrm{Rh}}$ & $\mathrm{O}$ & interstitial & total & Figure \\
\hline$\overline{\mathrm{LSDA}}$ & FM-M & 0 & 2.64 & & 0.37 & 0.13 & 0.16 & 4.00 & Fig. 1(b) \\
\hline LSDA & AF-M & 106 & \pm 2.63 & & 0 & \pm 0.12 & 0 & 0 & \\
\hline$\overline{\mathrm{LSDA}+\mathrm{U}}$ & FM-M & 0 & 2.85 & & 0.23 & 0.13 & 0.15 & 4.00 & Fig. 2(b) \\
\hline $\mathrm{LSDA}+\mathrm{U}$ & AF-M & 13 & \pm 2.87 & & 0 & \pm 0.11 & 0 & 0 & \\
\hline$\overline{\mathrm{LSDA}+\mathrm{U}+\mathrm{SOC}}$ & FM-I & 0 & 2.72 & 1.69 & 0.54 & 0.09 & 0.18 & 5.69 & Fig. 3 \\
\hline $\mathrm{LSDA}+\mathrm{U}+\mathrm{SOC}$ & AF-I & 70 & $2.73,-2.53$ & $1.69,-1.70$ & 0.55 & $0.09,-0.01$ & 0.07 & 1.00 & \\
\hline
\end{tabular}

\section{MODEL OF MAGNETISM}

In order to explain both the experimentally observed and theoretically (computationally) confirmed weak intrachain $\mathrm{AF}\left(T_{\mathrm{N}}=12 \mathrm{~K}\right)$ in $\mathrm{Ca}_{3} \mathrm{FeRhO}_{6}$ and relatively strong $\mathrm{FM}\left(T_{\mathrm{C}}=90 \mathrm{~K}\right)$ in $\mathrm{Ca}_{3} \mathrm{CoRhO}_{6}$, we propose the following model. Starting from our finding that $\mathrm{Ca}_{3} \mathrm{FeRhO}_{6}$ has the $\mathrm{HS} \mathrm{Fe}^{3+}$ and $\mathrm{LS} \mathrm{Rh}^{3+}$ (nonmagnetic $t_{2 g}^{6}$ ) valence state, a charge fluctuation will create the $\mathrm{Fe}^{2+} / \mathrm{Rh}^{4+}$ pair rather than $\mathrm{Fe}^{4+} / \mathrm{Rh}^{2+}$, since it is easier to stabilize a $\mathrm{Rh}^{4+}$ rather than an $\mathrm{Fe}^{4+}$. In Fig. 4(a), we sketch the normal superexchange mechanism $\mathrm{Fe}^{3+}-\left(\mathrm{O}^{2-}\right)-\mathrm{Rh}^{3+}-\left(\mathrm{O}^{2-}\right)-\mathrm{Fe}^{3+}$ via the intermediate non-magnetic $\mathrm{Rh}^{3+}$ (in the figure the $\mathrm{O}^{2-}$ is omitted for clarity). Since the $\mathrm{HS} \mathrm{Fe}{ }^{3+}$ has a closed up-spin $3 d$ shell, only the down-spin $\mathrm{Rh}^{3+}$ electron [e.g., the $a_{1 \mathrm{~g}}$ $\left.\left(3 z^{2}-r^{2}\right)\right]$ can make a virtual excitation, which naturally explains the intra-chain $\mathrm{AF}$, with a low $T_{\mathrm{N}}$ due to the large Fe-Fe distance of $5.39 \AA$. In fact, this result is a direct analog of the first Goodenough-Kanamori-Anderson rule. ${ }^{46}$

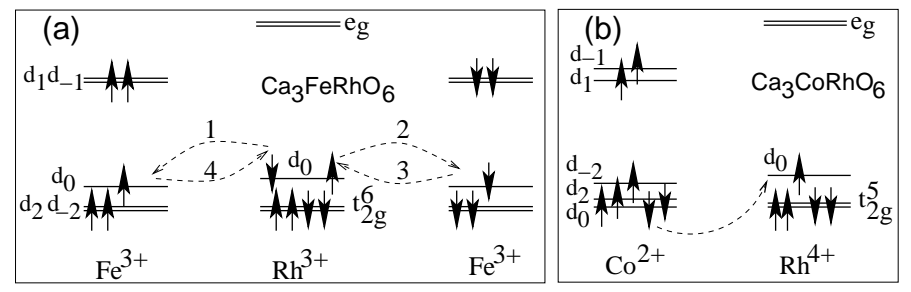

FIG. 4: Model of the intra-chain AF in $\mathrm{Ca}_{3} \mathrm{FeRhO}_{6}$ (a) and of the intra-chain FM in $\mathrm{Ca}_{3} \mathrm{CoRhO}_{6}$ (b).

$\mathrm{Ca}_{3} \mathrm{CoRhO}_{6}$ in contrast, has the $\mathrm{HS} \mathrm{Co}^{2+}$ and LS $\mathrm{Rh}^{4+}\left(t_{2 g}^{5}, S=1 / 2\right)$ valence state. We therefore consider the $\mathrm{Co}^{3+} / \mathrm{Rh}^{3+}$ charge fluctuation (rather than the $\mathrm{Co}^{1+} / \mathrm{Rh}^{5+}$ with a rarely observed $\mathrm{Co}^{1+}$ state) for the virtual excitation path $\mathrm{Co}^{2+}-\left(\mathrm{O}^{2-}\right)-\mathrm{Rh}^{4+}$. Since $\mathrm{Rh}^{4+}$ has a large $t_{2 g}-e_{g} \mathrm{CF}$ splitting which makes the empty $e_{g}$ states to be costly to reach, it will be the $t_{2 g}^{5}$ states which will allow for an electron transfer to the empty down-spin $a_{1 g}$ orbital, as shown in Fig. 4(b). If the $\mathrm{Co}^{2+}$ and $\mathrm{Rh}^{4+}$ have a FM alignment, the down-spin $\mathrm{Co}^{2+} a_{1 g}\left(d_{0}\right)$ electron will do so; otherwise (in the AF $\mathrm{Co}^{2+} / \mathrm{Rh}^{4+}$ alignment) the spin-up $a_{1 g}$ electron will do, but obviously with a higher energy cost than the former by 3 times Hund's exchange energy of the Co ion. Thus the $\mathrm{Co}^{2+}$ and $\mathrm{Rh}^{4+}$ strongly prefer the FM alignment, being an analog of the second Goodenough-KanamoriAnderson rule. ${ }^{46}$ In addition to the spin-triplet oxygenhole mediated intra-chain FM ordering present in both $\mathrm{Ca}_{3} \mathrm{CoRhO}_{6}$ and $\mathrm{Ca}_{3} \mathrm{Co}_{2} \mathrm{O}_{6},{ }_{23}$ this mechanism accounts for a higher $T_{\mathrm{C}}$ in the former than in the latter. Note that if $\mathrm{Ca}_{3} \mathrm{CoRhO}_{6}$ had the HS $\mathrm{Co}^{3+}$ and LS non-magnetic $\mathrm{Rh}^{3+}$ valence states, as in the case of $\mathrm{Ca}_{3} \mathrm{Co}_{2} \mathrm{O}_{6}$ with the HS trigonal $\mathrm{Co}^{3+}$ and LS non-magnetic octahedral $\mathrm{Co}^{3+}$, its $T_{\mathrm{C}}$ would be lower than that of $\mathrm{Ca}_{3} \mathrm{Co}_{2} \mathrm{O}_{6}$ due to a longer distance between the magnetic $\mathrm{HS} \mathrm{Co}^{3+}$ ions in the former than latter.

\section{CONCLUSION}

In summary, we have performed systematic LSDA and $\mathrm{LSDA}+\mathrm{U}$ calculations with the inclusion of the spinorbit coupling for the new quasi-one-dimensional spinchain materials $\mathrm{Ca}_{3} \mathrm{FeRhO}_{6}$ and $\mathrm{Ca}_{3} \mathrm{CoRhO}_{6}$. We conclude that $\mathrm{Ca}_{3} \mathrm{CoRhO}_{6}$ is a Mott insulator with the high-spin $\mathrm{Co}^{2+}$ and low-spin $\mathrm{Rh}^{4+}$ configurations, and that the correlated insulator $\mathrm{Ca}_{3} \mathrm{FeRhO}_{6}$ has the closedshell high-spin $\mathrm{Fe}^{3+}$ and low-spin $\mathrm{Rh}^{3+}$. We predict that $\mathrm{Ca}_{3} \mathrm{CoRhO}_{6}$ has a very large orbital moment at the $\mathrm{Co}^{2+}$ site, which also explains the strong magnetocrystalline anisotropy and the highly Ising-spin like behavior. The inclusion of the spin-orbit coupling in the present $\mathrm{LSDA}+\mathrm{U}$ calculations is also crucial to obtain the insulating state. This highlights the importance of the spin-orbit coupling in (nearly) degenerate correlated sys- 
tems. In contrast, $\mathrm{Ca}_{3} \mathrm{FeRhO}_{6}$ has spin-only moments and behaves like a Heisenberg spin-chain system. Moreover, our calculations reproduce the relatively strong ferromagnetic intra-chain coupling in $\mathrm{Ca}_{3} \mathrm{CoRhO}_{6}$ but the weak, presumably antiferromagnetic one in $\mathrm{Ca}_{3} \mathrm{FeRhO}_{6}$, and we propose a model to explain the contrasting mag- netism.

We thank M. W. Haverkort and T. Burnus for valuable discussions. This work is supported by the Deutsche Forschungsgemeinschaft through SFB 608 and by the European project COMEPHS.
1 H. Fjellvåg, E. Gulbrandsen, S. Aasland, A. Olsen, and B. Hauback, J. Solid State Chem. 124, 190 (1996).

2 S. Aasland, H. Fjellvåg, and B. Hauback, Solid State Commun. 101, 187 (1997).

3 H. Kageyama, K. Yoshimura, K. Kosuge, H. Mitamura, and T. Goto, J. Phys. Soc. Jpn, 66, 1607 (1997).

4 A. Maignan, C. Michel, A. C. Masset, C. Martin, and B. Raveau, Eur. Phys. J. B 15, 657 (2000).

${ }^{5}$ H. Kageyama, K. Yoshimura, K. Kosuge, M. Azuma, M. Takano, H. Mitamura, and T. Goto, J. Phys. Soc. Jpn, 66, 3996 (1997).

${ }^{6}$ B. Martínez, V. Laukhin, M. Hernando, J. Fontcuberta, M. Parras, and J. M. González-Calbet, Phys. Rev. B 64, 012417 (2001).

7 B. Raquet, M. N. Baibich, J. M. Broto, H. Rakoto, S. Lambert, and A. Maignan, Phys. Rev. B 65, 104442 (2002).

8 S. Rayaprol, K. Sengupta, and E. V. Sampathkumaran, Solid State Commun. 128, 79 (2003).

9 V. Hardy, S. Lambert, M. R. Lees, and D. McK. Paul, Phys. Rev. B 68, 014424 (2003).

10 A. Maignan, S. Hébert, C. Martin, and D. Flahaut, Mater. Sci. Eng., B 104, 121 (2003).

11 A. Maignan, V. Hardy, S. Hébert, M. Drillon, M. R. Lees, O. Petrenko, D. Mc K. Paul, and D. Khomskii, J. Mater. Chem. 14, 1231 (2004).

12 E. V. Sampathkumaran, N. Fujiwara, S. Rayaprol, P. K. Madhu, and Y. Uwatoko, Phys. Rev. B 70, 014437 (2004).

13 D. Flahaut, A. Maignan, S. Hébert, C. Martin, R. Retoux, and V. Hardy, Phys. Rev. B 70, 094418 (2004).

14 T. Sekimoto, S. Noguchi, and T. Ischida, J. Phys. Soc. Jpn. 73, 3217 (2004).

15 V. Hardy, D. Flahaut, M. R. Lees, and O. A. Petrenko, Phys. Rev. B 70, 214439 (2004).

16 K. Takubo, T. Mizokawa, S. Hirata, J.-Y. Son, A. Fujimori, D. Topwal, D. D. Sarma, S. Rayaprol, and E.-V. Sampathkumaran, Phys. Rev. B 71, 073406 (2005).

17 M.-H. Whangbo, D. Dai, H.-J. Koo, and S. Jobic, Solid State Commun. 125, 413 (2003).

18 R. Vidya, P. Ravindran, H. Fjellvåg, A. Kjekshus, and O. Eriksson, Phys. Rev. Lett. 91, 186404 (2003).

19 V. Eyert, C. Laschinger, T. Kopp, and R. Frésard, Chem. Phys. Lett. 385, 249 (2004).

20 R. Frésard, C. Laschinger, T. Kopp, and V. Eyert, Phys. Rev. B 69, 140405(R) (2004).

21 D. Dai and M.-H. Whangbo, Inorg. Chem. 44, 4407 (2005).

22 A. Villesuzanne and M.-H. Whangbo, Inorg. Chem. 44, 6339 (2005).

${ }^{23}$ H. Wu, M. W. Haverkort, Z. Hu, D. I. Khomskii, and L. H. Tjeng, Phys. Rev. Lett. 95, 186401 (2005).

24 Y. B. Kudasov, Phys. Rev. Lett. 96, 027212 (2006).

25 S. Niitaka, H. Kageyama, M. Kato, K. Yoshimura, and K. Kosuge, J. Solid State Chem. 146, 137 (1999).

26 S. Niitaka, H. Kageyama, K. Yoshimura, K. Kosuge, S.
Kawano, N. Aso, A. Mitsuda, H. Mitamura, and T. Goto, J. Phys. Soc. Jpn. 70, 1222 (2001).

27 S. Niitaka, K. Yoshimura, K. Kosuge, M. Nishi, and K. Kakurai, Phys. Rev. Lett. 87, 177202 (2001).

28 E. V. Sampathkumaran and A. Niazi, Phys. Rev. B 65, 180401(R) (2002).

29 M. J. Davis, M. D. Smith, and H-C. zur Loye, J. Solid State Chem. 173, 122 (2003).

30 M. Loewenhaupt, W. Schäfer, A. Niazi, and E. V. Sampathkumaran, Europhys. Lett. 63, 374 (2003).

31 S. Niitaka, K. Yoshimura, K. Kosuge, K. Mibu, H. Mitamura, and T. Goto, J. Magn. Magn. Mater. 260, 48 (2003).

32 V. Eyert, U. Schwingenschlögl, C. Hackenberger, T. Kopp, R. Frésard, and U. Eckern, cond-mat/0509374.

33 V. I. Anisimov, J. Zaanen, and O. K. Andersen, Phys. Rev. B 44, 943 (1991).

34 V. I. Anisimov, I. V. Solovyev, M. A. Korotin, M. T. Czyżyk, and G. A. Sawatzky, Phys. Rev. B 48, 16929 (1993).

35 P. Blaha, K. Schwarz, G. K. H. Madsen, D. Kvasnicka, and J. Luitz, http://www.wien2k.at

36 Actually in both $\mathrm{Ca}_{3} \mathrm{FeRhO}_{6}$ and $\mathrm{Ca}_{3} \mathrm{CoRhO}_{6}$ the octahedral $\mathrm{Rh}$ feels a trigonal crystal field. However, the $a_{1 g}$ singlet split off from the $t_{2 g}$ manifold lies above the $e_{g}^{\pi}$ doublet only by about $200(230) \mathrm{meV}$ in $\mathrm{Ca}_{3} \mathrm{FeRhO}_{6}$ $\left(\mathrm{Ca}_{3} \mathrm{CoRhO}_{6}\right)$, being much less than the cubic $t_{2 g}-e_{g}$ splitting of about $3 \mathrm{eV}$. Therefore, we keep to use in this work the conventional orbital index $t_{2 g}$.

37 I. V. Solovyev, A. I. Liechtenstein, and K. Terakura, Phys. Rev. Lett. 80, 5758 (1998).

38 J. Kuneš, H. Rosner, D. Kasinathan, C. O. Rodriguez, and W. E. Pickett, Phys. Rev. B 68, 115101 (2003).

39 I. I. Mazin and V. I. Anisimov, Phys. Rev. B 55, 12822 (1997).

40 I. Leonov, A. N. Yaresko, V. N. Antonov, M. A. Korotin, and V. I. Anisimov, Phys. Rev. Lett. 93, 146404 (2004).

41 H.-T. Jeng, G. Y. Guo, and D. J. Huang, Phys. Rev. Lett. 93, 156403 (2004).

42 M. Cococcioni and S. de Gironcoli, Phys. Rev. B 71, 035105 (2005).

43 Using LSDA+U+SOC calculations for two configuration states of the high-spin $\mathrm{Co}^{2+}$ ions in $\mathrm{Ca}_{3} \mathrm{CoRhO}_{6}$, i.e. the minority-spin $d_{0} d_{2}$ (the ground state, see more in the main text) and $d_{0} d_{-2}$ (a metastable state), we obtained the total-energy difference of $142 \mathrm{meV} / \mathrm{Co}$ between them, which is two times the SOC constant. Then the SOC constant is calculated to be 71 $\mathrm{meV}$. This is a commonly accepted value and agrees well with a Hartree-Fock value of $66 \mathrm{meV}$ (M. W. Haverkort, PhD thesis, University of Cologne, 2005; http://xxx.lanl.gov/abs/cond-mat/0505214).

44 J. Zaanen, G. A. Sawatzky, and J. W. Allen, Phys. Rev. Lett. 55, 418 (1985). 
$45 \mathrm{Z}$. Hu et al., to be published; see also the presentation TT 25.96 at the Spring Meeting of The German Physical Society, March 2006 (website: http://www.dpg-tagung.de/archive/2006/dresden/tt25.pdf). Note also that our previous prediction of a huge orbital moment in the isostructural $\mathrm{Ca}_{3} \mathrm{Co}_{2} \mathrm{O}_{6}$ (Ref. 23) was already confirmed experimentally [T. Burnus et al., Phys. Rev. B 74, 245111 (2006)].

46 J. B. Goodenough, in Magnetism and the Chemical Bond, (Interscience Publishers, New York-London, 1963). 